\title{
Dominance Attributions Following Damage to the Ventromedial Prefrontal Cortex
}

\author{
Matthew S. Karafin ${ }^{1}$, Daniel Tranel ${ }^{1}$, and Ralph Adolphs ${ }^{2}$
}

\begin{abstract}
Damage to the human ventromedial prefrontal cortex (VM) can result in dramatic and maladaptive changes in social behavior despite preservation of most other cognitive abilities. One important aspect of social cognition is the ability to detect social dominance, a process of attributing from particular social signals another person's relative standing in the social world. To test the role of the VM in making attributions of social dominance, we designed two experiments: one requiring dominance judgments from static pictures of faces, the second requiring dominance judgments from film clips. We tested three demographically matched groups of subjects: subjects with focal lesions in the VM $(n=$ $15)$, brain-damaged comparison subjects with lesions excluding the VM $(n=11)$, and a reference group of normal in-
\end{abstract}

\section{INTRODUCTION}

What is the difference between the CEO of a major corporation and a junior executive? Salary, authority, and work obligations encompass correlated differences that are captured in the construct of dominance. Dominance is signaled by stereotypical differences in age, gender, ethnicity, facial expression, posture, and attire; and in turn dictates stereotypical patterns of social interaction. It is well established that both human and nonhuman primates have hierarchically structured societies, such that individuals within a society can be identified by their dominance relationships to others. Dominance is closely correlated with ecologically important behavioral variables: for example, men who are perceived to be socially dominant also tend to be perceived as more attractive and tend to have sexual encounters at an earlier age (Mazur, Halpern, \& Uldry, 1994; Reis et al., 1982).

The accurate and rapid attribution of dominance by conspecifics is a necessary component of social cognition. "Seeing" dominance, however, draws on numerous cues, including visual information from face, posture, attire, and visible behavior, as well as background knowledge about the person. Previous studies using human

\footnotetext{
${ }^{1}$ University of Iowa, ${ }^{2}$ California Institute of Technology
}

dividuals with no history of neurological disease $(n=32)$. Contrary to our expectation, we found that subjects with VM lesions gave dominance judgments on both tasks that did not differ significantly from those given by the other groups. Despite their grossly normal performance, however, subjects with VM lesions showed more subtle impairments specifically when judging static faces: They were less discriminative in their dominance judgments, and did not appear to make normal use of gender and age of the faces in forming their judgments. The findings suggest that, in the laboratory tasks we used, damage to the VM does not necessarily impair judgments of social dominance, although it appears to result in alterations in strategy that might translate into behavioral impairments in real life.

subjects have shown that multiple visual cues can influence social dominance attributions. In faces, older age, male gender of the face, lower brow position, lack of a smile, and intense emotional expressions such as anger, are all associated with higher perceived dominance (Hess, Blairy, \& Kleck, 2000; Senior et al., 1999; Keating, 1985; Keating, Mazur, Segall, 1977; Keating, Mazur, \& Segall, 1981). Indeed, social dominance can be signaled reliably on the basis of some of these cues in schematic face drawings (Senior et al., 1999). All of these dominance associations have been observed already in school-age children, indicating that the socialization involving the attribution of dominance gestures begins early in life (Keating \& Bai, 1986) or that such attributions may in fact be partly innate. Beyond the face, body posture, tone of voice, clothing style, as well as background personal information, all communicate something about our place in the social hierarchy (Brothers, 1997).

A key candidate brain region for processing dominance information is the ventromedial prefrontal cortex (VM). The VM region encompasses ventral and medial sectors of the prefrontal cortex, and includes Brodmann's areas 11, 12, 13, 25, 32, and 10 (Bechara, Damasio, \& Damasio, 2000). Ever since the classic case of Phineas Gage, damage to this region has been associated with changes in social behavior in both human 
and nonhuman primates, despite intact cognitive skills such as language, memory, and attention (Barrash, Tranel, \& Anderson, 2000; Damasio, 1994; Raleigh \& Steklis, 1981; Deutsch, Kling, \& Steklis, 1979). In humans, these changes include blunted emotional experience, apathy, low emotional expressiveness, poor judgement, social inappropriateness and isolation, lack of planning, and lack of insight (Barrash et al., 2000).

We have had the opportunity to observe impairments putatively related to abnormal judgments of social dominance in such patients in our own laboratory. One of our patients with bilateral VM damage has been tested in our laboratory on numerous occasions. Often, the testing sessions have involved members of our staff who had vastly different standing in the institutional hierarchy: for example, the head of the department, a postdoctoral student, and an undergraduate summer intern. The patient appeared fairly oblivious to this hierarchy: He would interrupt the department head or the intern with equal frequency, make jokes with any of the parties with equal regularity, and attend to and engage any of the three staff with equal proclivity. This behavior is quite different from that of, for example, one of our aphasic patients, who clearly perceived the dissimilarity of rank among the staff persons and behaved accordingly, for instance, by devoting disproportionate attention to the department head, ignoring the intern, and making more eye contact with the department head.

Despite the importance of the VM region to socioemotional processing, and despite the anecdotal behavioral observations indicating impaired processing of social dominance information, humans with VM lesions are often able to provide relatively normal cognitive judgments, including social judgments, provided that these judgments are made in the abstract, or in the laboratory, rather than in the real world. For instance, Saver and Damasio (1991) found that such subjects failed to show obvious impairments when judging abstract moral dilemmas, despite their patently impaired ability to use such judgments to guide their behavior in real life. Nonetheless, it may be possible to reveal an impairment even in the laboratory, provided that the social information is not entirely explicit: Stone, BaronCohen, and Knight (1998) found that patients with VM damage were unable to recognize contextual faux pas; a similar study concluded that VM patients have difficulty interpreting sarcastic comments (Shamay, AharonPeretz, Berger, \& Tomer, 2001) and show a diminished implicit sex bias (Milne \& Grafman, 2001). These findings are consistent with results from functional imaging, which has reported activation of the VM during tasks requiring prediction of the motivational state of another person (Goel, Grafman, Sadato, \& Hallett, 1995) or in moral judgment (Greene, Sommerville, Nystrom, Darley, \& Cohen, 2001).

Based on this background, we hypothesized that the VM would be involved in judging social dominance, but that its role would be critical only when such judgments could not be compensated for by reasoning from explicit cues. We thus designed two tasks: one using static pictures of faces that provided less explicit social information, and a second using videos that provided rich and more explicit social cues. Although subjects with VM damage performed broadly normally on both tasks, they showed a lowered sensitivity to dominance cues in the face task, but not in the video task. We suggest such patients may generate a grossly normal performance on many tasks of dominance judgments, but that they use abnormal strategies in doing so-strategies that, we believe, are likely to contribute to their impaired social behavior in the real world.

\section{RESULTS}

\section{Background Neuropsychology and Demographics}

We tested three subject groups: those with focal damage to the ventromedial prefrontal cortex (VM; $n=15$; Figure 1), a brain-damaged comparison group with focal damage sparing the VM (BDC; $n=11)$, and a reference group of normal individuals with no neurological or psychiatric history (NC; $n=32$ ). Subjects in all three groups had similar ages, sex ratios, mean education, and mean scores on the Benton Facial Recognition Task, a measure of basic visual perception (Table 1). Although groups did not differ on any of these variables, braindamaged comparison subjects did have a lower mean verbal IQ than did normal subjects (Tukey $t$ test, $p<$ $.0005)$, likely a consequence of the fact that the majority of brain-damaged comparison subjects had lesions in the left hemisphere.

\section{Experiment 1: Face Task}

We first showed subjects static faces and asked them to rate dominance, as well as other control questions. Although we did not measure the time taken to produce the ratings, our informal impression was that VM subjects in fact took somewhat less time to make dominance judgments than did subjects in the other two groups. Subjects in all three groups rated all the faces as unfamiliar. Overall, the mean dominance judgments made by NC subjects, BDC subjects, and VM subjects were very similar $[F(2,55)=0.306, n s]$. A similar pattern of essentially identical judgments was found when subjects were asked to judge the age of the stimuli in a control task.

We next divided the 80 faces into subsets according to their gender (man or woman), age (old or young), facial expression (smiling or not smiling), and type of clothing (professional or casual) (cf. Table 2), and examined possible dominance contrasts between these subsets. These analyses revealed that normal subjects give differential dominance judgments for all of these factors. 
Figure 1. Distribution of lesions in VM subjects. The lesions of all subjects with damage in the VM were mapped onto a common reference brain and reconstructed. Three-dimensional rendering of the brain, and of representative coronal sections, are shown. Color encodes the number of lesions at a given location.

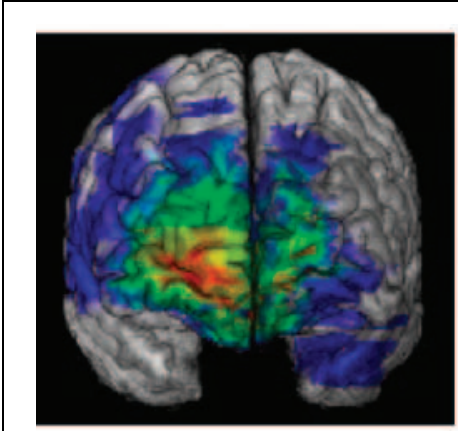

Number of Lesions:

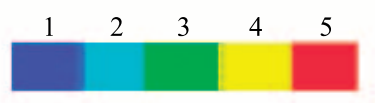

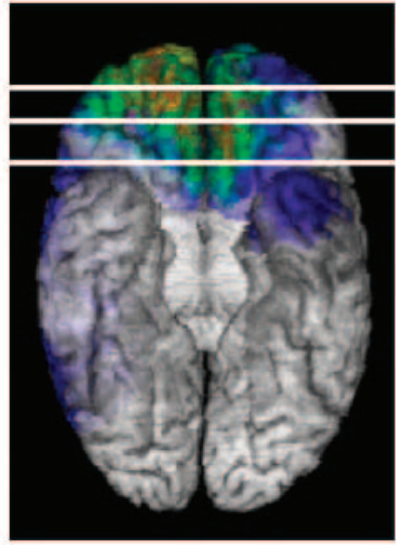
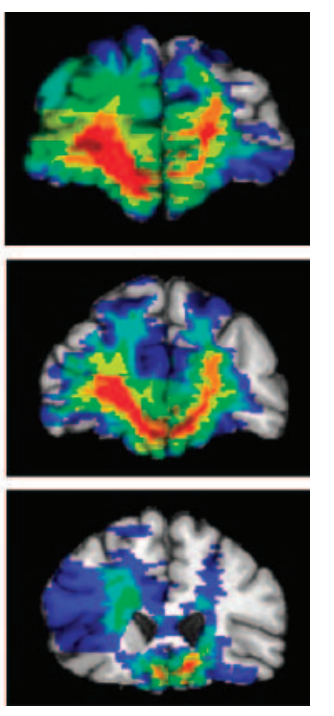

Specifically, normal subjects found male faces to be more dominant than female faces $(t=5.5, p=$ .0001 ), old (older than 55) faces to be more dominant than young (younger than 35) faces $(t=5.8, p=$ .0001), nonsmiling faces to be more dominant than smiling faces $(t=8.7, p=.0001)$, and professionally dressed individuals to be more dominant than casually dressed individuals $(t=8.4, p=.0001)$. Normal subjects also judged older men to be more dominant than younger men $(t=8.1, p<.0005)$, and, somewhat counterintuitively, younger women to be more dominant than older women $(t=3, p<.005$; all $p$ s uncorrected). We also analyzed the faces using normative judgments of face masculinity and anger (cf. Methods). We took the five faces at the extremes of each of these ratings and contrasted their dominance judgments. All subject groups found masculine faces to be more dominant than feminine faces $(p s<.01)$. When we conducted this analysis separately for male and female faces, all three subject groups judged male faces rated high on masculinity to look more dominant than male faces rated low on masculinity, but no such difference in dominance as a function of perceived masculinity existed for female faces (male faces: $p$ s < .0001; female faces: $p s>.05)$. Likewise, all groups judged angry faces to look more dominant than nonangry faces $(p s<.01)$, but, as for masculinity judgments, this effect held only for male faces (male faces: $p s<.0001$; female faces: ps $>.05)$.

When the mean dominance ratings given to each face were examined in more detail, a specific difference emerged between the NC and VM groups. Although their mean dominance ratings across all the faces did not differ (Figure 2B), NCs used a larger range of dominance ratings than did VM subjects, as evidenced by the larger $S D$ of dominance ratings across faces (Figure 2B) and the larger range of dominance ratings used by each individual subject (Figure 2C). Whereas the mean dominance ratings for the faces were highly correlated among the VM and NC groups $(r=.624$; $p<.0001)$, their range differed $(t=2.2, p<.05)$. The difference in range of dominance ratings between NC and BDC groups, by contrast, was not significant $(t=$ $1.09, n s)$ and the abnormal range use we saw in the VM group was not a general tendency in using a narrower range, as the ranges of the judgments of age produced by the two groups were identical.

We next looked further at the influence of the above factors on dominance judgments, by calculating contrast scores. We took the mean dominance ratings for

Table 1. Subject Demographics for Each of the Three Subject Groups

\begin{tabular}{lccccc}
\hline Group & Gender $(M / F)$ & Age $(S D)$ & Education (SD), years & VIQ (SD) & Benton (SD) \\
\hline NC $(n=32)$ & $16 / 16$ & $54.2(9)$ & $15(2.1)$ & $110.3(7.2)$ & $47.3(3.7)$ \\
VM $(n=15)$ & $8 / 7$ & $57(10.5)$ & $13.2(2.3)$ & $109.7(13.8)$ & $44.9(5)$ \\
BDC $(n=11)$ & $6 / 5$ & $58.9(17.7)$ & $13.8(2.3)$ & $92.4(13.3)$ & $45.6(3.6)$ \\
\hline
\end{tabular}

VIQ = verbal IQ from the Wechsler Adult Intelligence Scale; Benton $=$ corrected score on the Benton Facial Discrimination Task, a measure of visuoperceptual ability. 
Table 2. Face Stimuli Characteristics

\begin{tabular}{lcccc}
\hline Face Age & $20-35$ & $35-55$ & $55+$ & Total \\
\hline Smiling (M/F) & $8(4 / 4)$ & $20(8 / 12)$ & $9(5 / 4)$ & $37(17 / 20)$ \\
Not smiling (M/F) & $9(5 / 4)$ & $20(8 / 12)$ & $14(7 / 7)$ & $43(20 / 23)$ \\
Total (M/F) & $17(9 / 8)$ & $40(16 / 24)$ & $23(12 / 11)$ & $80(37 / 43)$ \\
\hline
\end{tabular}

Gender proportions and number of smiling faces, broken down as a function of the rated age of the faces.

faces of a particular subcategory (e.g., male faces) and subtracted that mean from the mean dominance ratings of its opposing subcategory (e.g., female faces). Figure 3 shows the mean contrast scores across various categories: VM subjects produced contrast scores that were consistently smaller than those of NCs. An ANOVA found significant group differences in the contrast scores for old-young faces $[F(2,55)=3.9, p<.05]$ and for male-female faces $[F(2,55)=7.6, p<.005]$. In all these three cases, post hoc Tukey tests showed that these group differences resulted from significant differences between VM subjects and normal subjects $(p s<.03)$. Brain-damaged comparison subjects did not differ significantly from normal subjects for any of these contrasts.

\section{Experiment 2: Video Task}

In Experiment 2, we showed subjects two videos and asked them to rank quantitatively the dominance of four actors in each video by marking an analog line (cf. Methods and Figure 5). Prior to viewing the video, subjects were shown static snapshots of each actor's face and asked to rate dominance as in Experiment 1; after viewing the video, subjects were questioned about the cues they believed were most important in making their dominance judgments. As in the case of the faces, while we did not quantitatively measure the time taken to rate the stimuli, our anecdotal impression was that the VM patients gave their dominance ratings no more slowly than did the two other subject groups and, if anything, gave them somewhat faster.

For the two video clips that we showed, we first analyzed subjects' estimation of each actor's age, familiarity with each actor, and general familiarity with the video clip. All subjects judged age similarly [Video 1: $F(2,55)<2.7$, ps $>.07$ for all four actors; Video 2 : $F(2,55)<1.7, p s>.19$ for all four actors]. Not surprisingly, several subjects were familiar with the actors in the video clips, but subject groups did not differ in their familiarity with the actors. To verify that familiarity with the actor(s) would not influence dominance judgments, we examined separately the mean dominance judgments given by subjects familiar or unfamiliar with the actor. For both videos, we found that subjects familiar with the actor did not differ in their judgments from those that were unfamiliar with the actor. There was also no effect of the order in which the two video clips were shown. All subjects comprehended the videos equally well, producing mean correctness scores on the multiple-choice follow-up questionnaire that were around $75 \%$; there were no group differences on this measure.

The three subject groups did not differ in their judgments of dominance from viewing actors shown in the videos [Video 1: $F(2,55)<.217, p>.8$; Video 2: $F(2,55)<.847, p>.4]$. As one might expect, subjects changed their dominance ratings for an actor between the initial static viewing of the actor's face prior to the video, and after having seen the actor in the video. All subject groups also showed the same pattern of such changes. The data from the dominance ratings from Experiment 2 are summarized in Figure 4.

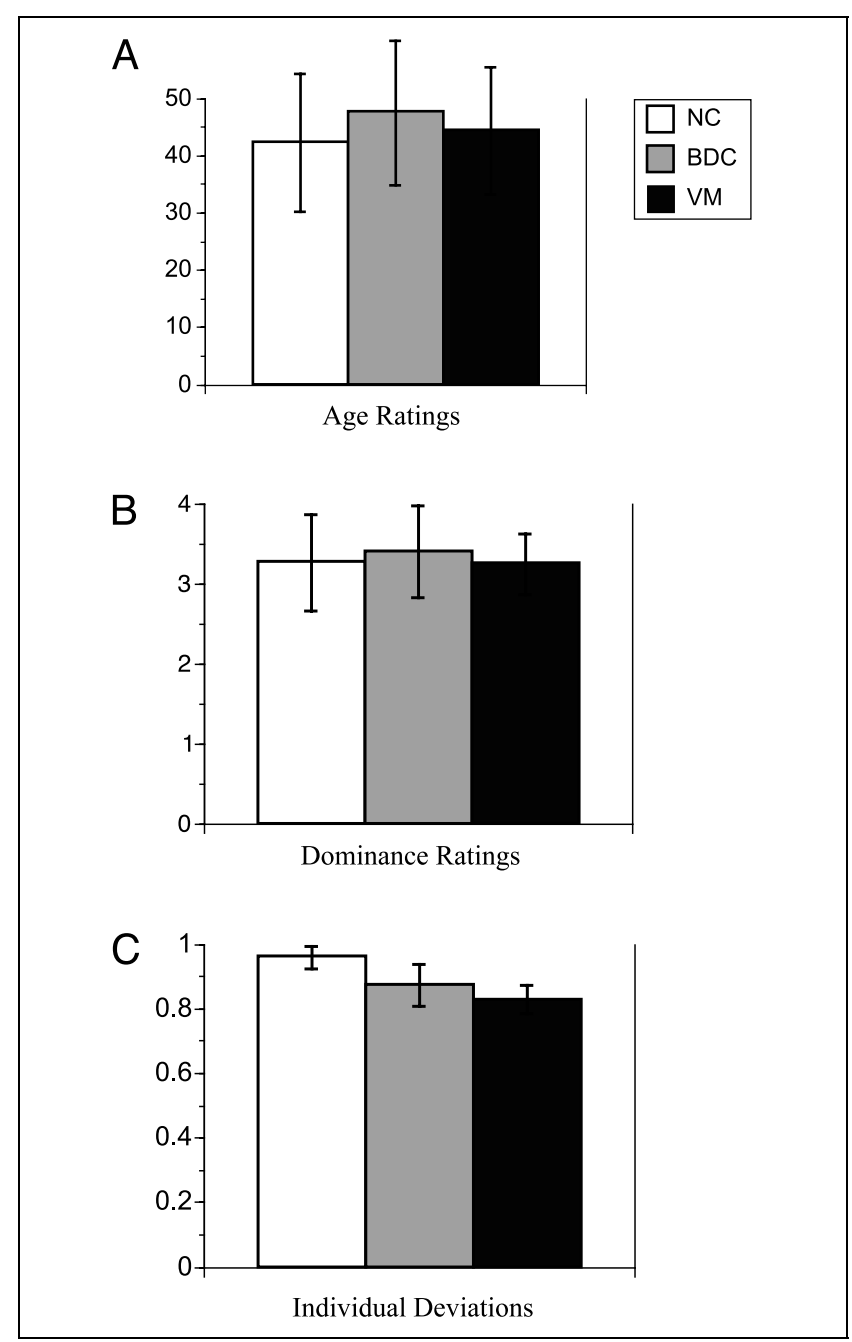

Figure 2. Experiment 1: ratings of faces. (A) Ratings of age (means and $S D$ ). (B) Ratings of dominance (means and $S D$ ). (C) Mean deviation of ratings for each individual subject (means and SEM); here the standard deviation was calculated across all the face stimuli for each individual subject, and individual subjects' SDs were then averaged within groups to yield the data shown in the plot. 
Figure 3. Experiment 1: dominance contrast ratings for faces. Each bar shows the mean (and SEM) for the absolute value of the difference in ratings between male and female faces, direct and averted gaze, smiling and nonsmiling expressions, and old and young faces. Normal subjects showed larger differences in their ratings of these dichotomizations than did subjects with VM lesions.

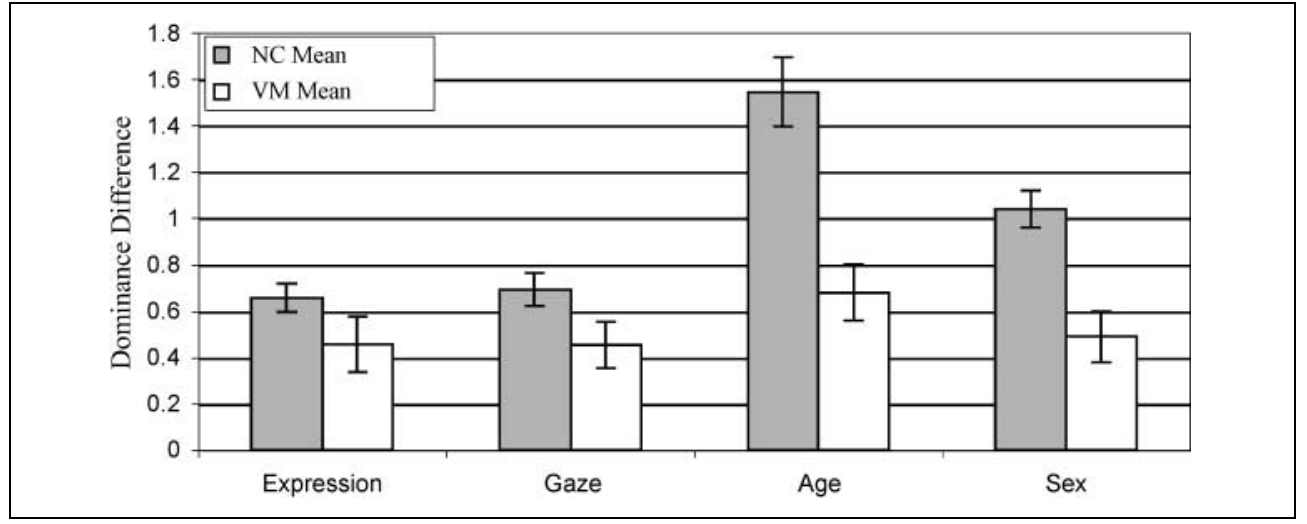

After they had seen the videos, subjects were asked about their reasons for producing their judgments. All subjects reported that the content of the video dialogue, the intonation of the actor's voice, and the time that an actor was on screen were the most important factors used when judging dominance for each movie. Subjects also reported that an actor's attire, their prior knowledge of the actor, and the name of the actor's character in the film had very little to do with their dominance judgments. Subject groups did not differ in the ratings they gave on any of the eight follow-up questions we asked (cf. Methods) $[F(2,55)<1.8, p s>.18]$.

\section{DISCUSSION}

We used static face stimuli and video clips to investigate dominance judgments. We found reliable judgments in normal participants, and some evidence for subtly abnormal judgments in subjects with damage to the VM.

In normal individuals, dominance judgments were produced reliably in both experiments, and reflected the use of socially relevant visual cues. The reliability with which normal subjects can make dominance judgments has been documented in prior studies (Keating, Mazur, Segall, 1981). Thus, people who appear dominant must have facial and behavioral features that make them appear dominant to most viewers. We found that certain specific features stereotypically signal dominance: Older faces were judged more dominant than younger faces, men more dominant than women, nonsmiling faces more dominant than smiling faces, and people with professional attire more dominant than those with casual attire. Furthermore, we found that these different sets of cues interact. One can thus envision a large set of cues-some probably innately specified, others culturally acquired-that are all considered together when viewers form dominance judgments about the person they see.

How the brain implements such a multidimensional evaluation is clearly a complex issue. At the level of visual processing, many of these different cues would be processed by partly distinct neuroanatomical regions. Yet each visual cue must be evaluated, and all the multiple cues must be integrated to produce the single dominance judgment that viewers make. Our hypothesis was that ventral and medial sectors of the prefrontal cortex perform just such a function. The extensive connections between temporal visual regions and the prefrontal cortex (Seltzer \& Pandya, 1989) provide for an effective source of perceptual input to this region of the brain, wherein one finds responses to a large range of visual stimuli in both monkeys (Hikosaka \& Watanabe, 2000) and humans (Taylor, Liberzon, \& Koeppe, 2000). While the prefrontal cortex participates in a widespread connectional network that permits the synthesis of emotional, mnemonic, attentional, and other cognitive functions (Barbas, 2000), ventral and medial sectors of this region have the most extensive connections with other brain structures involved in regulating emotional and social behaviors (Öngür \& Price, 2000; Damasio, 1996). It is in virtue of this architecture that the VM is a likely candidate to mediate between perception of socially significant visual stimuli and the triggering of emotional and cognitive processes that generate attributions of their social dominance.

In its strong form, our initial hypothesis that the VM is necessary for judging social dominance was refuted. Subjects with VM lesions were able to perform entirely normally in judging dominance from watching video clips (Figure 4). They also performed grossly normally when judging static pictures of people's faces. However, a closer examination revealed subtle abnormalities in their judgments-abnormalities that suggest altered processing of dominance information while nonetheless producing overall normal ratings of dominance.

Our preliminary interpretation of these findings is that the restricted range of dominance ratings, and the smaller contrast scores, that are generated by VM subjects, reflect an underlying insensitivity to variations in the stimuli (such as age and gender) that normally influence dominance judgments. However, the fact that the impairment is subtle, and the fact that it is not apparent when judging the video stimuli, suggests that VM 


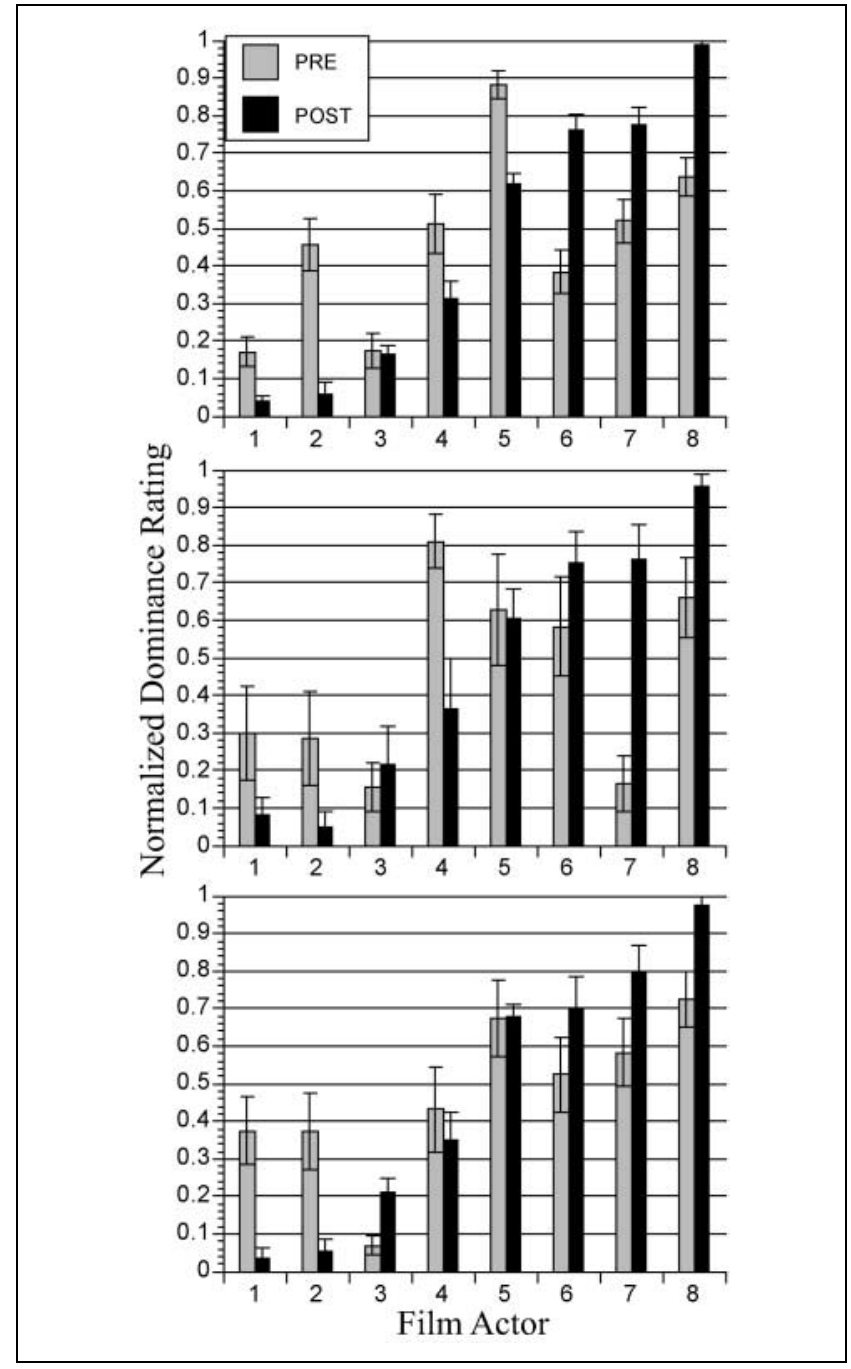

Figure 4. Experiment 2: pre- and postvideo ratings for the four actors shown in the two videos (eight actors total) (means and SEM). Each actor ( $x$-axis) is rank-ordered according to the mean NC post rankings of dominance that actor received. Top: NC; middle: BDC; bottom: VM.

subjects are able to make some, albeit more limited, use of social cues in order to figure out dominance judgments. We thus suggest that VM subjects are less sensitive to the social value of specific perceptual cues, rather than that they are entirely incapable of making social judgments, or have no concept of dominance. This interpretation explains the pattern of data as follows. When only sparse social cues are available, as was the case in our face task, or when subtle and fleeting social cues must be utilized in real time, as is the case in real life, the insensitivity translates into a compressed range of judgments, smaller contrast scores, or impaired real-world behavior. On the other hand, when a rich array of social cues are available in a stereotypical setting, as was the case with our video stimuli, the abundance of cues is sufficient to outweigh the insensitivity to them, and apparently normal judgments re- sult. Future studies could test these ideas directly, for instance, by parametrically altering the amount of social information present in the stimuli (e.g., creating a range that includes faces, body postures, context, dynamic information, and voices), and by constraining the processing time available (by presenting only short durations and/or measuring reaction times).

A final intriguing issue concerns the consequences of damage to the VM when acquired during development. Such developmental damage appears to damage not only one of the retrieval routes for social information, but also its acquisition in the first place. Consequently, subjects with developmental prefrontal cortex damage are not able either to guide normally their real-life social behavior (an impairment they share with patients who sustained VM damage in adulthood), or to make abstract social judgments when the information is provided explicitly (an impairment on which they differ from patients with adulthood VM lesions) (Anderson, Bechara, Damasio, Tranel, \& Damasio, 1999). We would thus expect that patients with such developmental damage would be more clearly impaired in their dominance judgments in our experiments, a prediction we are currently testing in ongoing studies.

\section{METHODS}

\section{Subjects}

We tested 15 subjects with focal ventromedial prefrontal cortex lesions (VM), 11 subjects with focal damage sparing the VM, mostly in the higher-order visual cortex (brain-damaged comparison subjects; BDC), and 32 age, education, and sex ratio matched normal comparison subjects (NC) without any history of neurological or psychiatric illness (Table 1). Figure 1 shows the distribution of lesions for the VM subjects. All subjects gave written informed consent for their participation, as approved by the Institutional Review Board of the University of Iowa.

\section{Face Judgment Task}

\section{Stimuli}

Our final task consisted of 80 digitized photographs of human faces, selected from over 200 initial stimuli in an extensive series of pilot studies. In the pilot studies, a stimulus was chosen if all normal subjects $(n=14)$

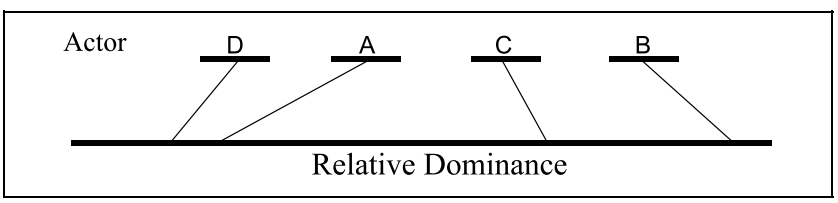

Figure 5. Experiment 2: snapshot of the dominance ranking task used for the actors in the videos. 
judged its dominance within 1 standard deviation of the pooled variance, and such that the age, sex, and expression of the faces were roughly evenly distributed. We chose faces that showed a minimum of emotional expression because we wanted to avoid the obvious cues that emotional expression might provide. Faces were selected from various media sources and showed black and white images of unfamiliar individuals that revealed only the person's face and neck. Stimuli were distributed homogeneously across gender, age, and facial expression (Table 2). Stimuli were shown self-paced, one at a time, in randomized order.

\section{Procedure}

Subjects were asked to make three judgments for each face, based solely on its appearance. The first judgment required subjects to rate dominance, using a scale from 1 to 5 . The dominance scale was described to subjects to be like a totem pole: The top of the pole ( 5 on our scale) represented individuals who tell other people what to do, and are obeyed by most people, the middle part of the pole ( 3 on the scale) represented "average" individuals who can give orders to people below them on the totem pole, but cannot direct the activities of those people who are higher on the pole, and the lowest part of the pole (a 1 on the scale) represented those who do not have any ability to influence the actions of others above them. The second judgment asked subjects to estimate the age of the person, in years. The third judgment asked subjects if the face looked familiar to them (yes/no).

\section{Characterization of Face Stimuli}

In order to further characterize our static face stimuli, a subset of our normal control population $(n=11)$ was asked to rate each face for its masculinity, and for the intensity of happiness, anger, and fear expressed. For the masculinity judgment, subjects rated the face on a scale of 1-10 and were informed that the scale did not represent a judgment of sex, but rather how masculine or feminine the face was, regardless of sex (i.e., a woman who looked masculine could be given a high number). All of the emotions were judged on Likert scales of $0-5$. A summary of these supplemental ratings can be found in Table 3. We found that, on average, male faces in this task were rated more masculine than female faces.
Moreover, there was a weak positive correlation between dominance judgments and masculinity in normal subjects $(r=.354, p<.001)$. We also found that our faces were generally devoid of emotion. Specifically, faces were generally rated as having minimal or no anger or fear, and happiness was only moderate for most of our face stimuli. Of the three rated emotions, only anger was found to be correlated with dominance $(r=.299, p<.01)$.

\section{Data Analysis for the Face Task}

For all analyses using the age of the face as a factor, the mean age judgment given by normal control subjects was used as the "correct" age for each face. Face familiarity was operationalized as the familiarity judgment that a subject gave, regardless of its accuracy (all the faces were in fact unfamiliar). Face sex (as opposed to the continuous masculinity ratings described above), however, was assigned dichotomously based on the objective properties of the face.

\section{Video Judgment Task}

\section{Video Stimuli}

The final task asked subjects to view two video clips derived from two different commercially available films. These clips were chosen from an initial sample of five based on a pilot study in eight normal subjects, such that the dominance ratings showed the least variation among subjects. The final clips were 5 and $8 \mathrm{~min}$ in duration, and depicted a social interaction between four or more individuals. Specifically, the first film involved a grouptherapy session taken from "One Flew Over the Cuckoo's Nest," and the second film involved a jury that was debating the facts of a trial taken from "Twelve Angry Men." Clips were converted to Quicktime movies and viewed full screen on a Macintosh monitor. Subjects wore noise-reduction headphones for the audio.

\section{Procedure}

First, subjects were given a preliminary task in which they viewed pictures of the faces of the four actors (face and neck only) simultaneously on the screen. These images were taken directly from the video clip that they were about to see, and each actor was assigned a letter

Table 3. Supplementary Characterization of Face Stimuli

\begin{tabular}{lcccc}
\hline Face Characteristic & Face Masculinity (SD) & Face Anger (SD) & Face Fear (SD) & Face Happiness (SD) \\
\hline Male faces (37) & $7.88(0.9)$ & $0.91(0.91)$ & $0.42(0.38)$ & $2.7(1.1)$ \\
Female faces (43) & $3.52(0.88)$ & $0.73(0.57)$ & $0.59(0.51)$ & $2.69(1.1)$ \\
\hline
\end{tabular}

The rated masculinity (cf. Methods), and intensity of anger, fear, and happiness, for the male and female faces. 
(A, B, C, D). Subjects were asked to make three judgments about each of the four faces shown on screen, prior to viewing the film clip: dominance, age, and familiarity. Age and familiarity were rated exactly as in the static face task (cf. above). Dominance, however, required subjects to rank the pictures by indicating their relative dominance on an analog line. Subjects were asked to imagine that the four people they saw would be socially interacting in a group, and to estimate solely from the face who would be the most dominant, second most dominant, and so on. Subjects placed a mark on a 10-cm line to indicate where they thought each actor fell on a spectrum from most to least dominant (Figure 5).

After completing the preliminary task for both videos, subjects were shown the video clips. To aid reference to the four actors whose dominance they were rating, subjects were shown the relevant four actors' faces on another nearby computer screen during the entire video presentation. Subjects were instructed to watch the video carefully and to learn as much about each of the four actors as they could. Additionally, subjects were told that they could watch the video a second time, but the second viewing could only occur immediately after the first, before giving any of the ratings. In other words, if the subject decided to start answering questions about the video clip, they were no longer able to view the video a second time. This procedure was repeated for the second video clip.

Following each video, subjects were given a 12 -item questionnaire and a 14-item multiple-choice test. The questionnaire, which was counterbalanced across subjects, involved ranking the four labeled actors seen in the video. The rankings were recorded in a manner identical to the dominance judgments of the preliminary task, and involved ranking the four actors on dominance, as well as other characteristics such as leadership and trustworthiness. Several of these ratings were highly correlated with dominance; here we focus only on the ratings given on dominance. The multiplechoice test was given as a control task, and asked questions that could only be answered correctly if the subject comprehended the video clip and remembered certain salient features about it. We also asked subjects how familiar they were with the movie from which the video clip was taken.

A final questionnaire was given immediately after the subject finished evaluating both of the movies. The final questionnaire aimed to assess the subject's opinion of what features of the video influenced them the most while making judgments about the actors onscreen. Specifically, subjects were asked to consider eight general characteristics present in both video clips: the overall content of the clip, the verbal intonation of the actor, the overall visual appearance of the actor, how the actor moved, their prior knowledge about the movie and/or the actor, the dress of the actor, the title of the actor in the movie, the amount of time the actor was on the screen. Subjects rated the importance of each of these variables on a scale from 1 to 9 .

\section{Data Analysis for the Video Task Rankings}

The relative rankings produced by analog markings on the $10-\mathrm{cm}$ line were quantified by measuring the location of the marks placed for each actor. These numerical values were then normalized by taking the relative distance of each actor's mark and dividing by the total range of the marks, in order to accommodate for variations in range produced by different subjects. This produced a normalized score between 0 and 1 .

\section{Acknowledgments}

We thank T. W. Buchanan for help with the statistical analyses, and R. Henson, K. Manzel, and J. Shultz for help with scheduling and testing of subjects.

Reprint requests should be sent to Ralph Adolphs, HSS 228-77, Caltech, Pasadena, CA 91125, or via e-mail: radolphs@hss. caltech.edu.

\section{REFERENCES}

Anderson, S. W., Bechara, A., Damasio, H., Tranel, D., \& Damasio, A. R. (1999). Impairment of social and moral behavior related to early damage in human prefrontal cortex. Nature Neuroscience, 2, 1032-1037.

Barbas, H. (2000). Connections underlying the synthesis of cognition, memory, and emotion in primate prefrontal cortices. Brain Research Bulletin, 52, 319-330.

Barrash, J., Tranel, D., \& Anderson, S. W. (2000). Acquired personality disturbances associated with bilateral damage to the ventromedial prefrontal region. Developmental Neuropsychology, 18, 355-381.

Bechara, A., Damasio, H., \& Damasio, A. R. (2000). Emotion, decision-making, and the orbitofrontal cortex. Cerebral Cortex, 10, 295-307.

Brothers, L. (1997). Friday's footprint. New York: Oxford University Press.

Damasio, A. R. (1994). Descartes' error: Emotion, reason, and the buman brain. New York: Grosset/Putnam.

Damasio, A. R. (1996). The somatic marker hypothesis and the possible functions of the prefrontal cortex. Philosophical Transactions of the Royal Society of London Series B, 351, 1413-1420.

Deutsch, R. D., Kling, A., \& Steklis, H. D. (1979). Influence of frontal lobe lesions on behavioral interactions in man. Research Communications in Psychology, Psychiatry and Behavior, 4, 415-431.

Goel, V., Grafman, J., Sadato, N., \& Hallett, M. (1995). Modeling other minds. NeuroReport, 6, 1741-1746.

Greene, J. D., Sommerville, R. B., Nystrom, L. E., Darley, J. M., \& Cohen, J. D. (2001). An fMRI investigation of emotional engagement in moral judgment. Science, 293, 2105-2107.

Hess, U., Blairy, S., Kleck, R. E. (2000). The influence of facial emotion displays, gender, and ethnicity on judgments of dominance and affiliation. Journal of Nonverbal Behavior, 24, 265-283.

Hikosaka, K., \& Watanabe, M. (2000). Delay activity of orbital and lateral prefrontal neurons of the monkey varying with different rewards. Cerebral Cortex, 10, 263-271. 
Keating, C. F. (1985). Gender and the physiognomy of dominance and attractiveness. Social Psychology Quarterly, $48,61-70$.

Keating, C. F., \& Bai, D. L. (1986). Children's attributions of social-dominance from facial cues. Child Development, 57, 1269-1276.

Keating, C. F., Mazur, A., \& Segall, M. H. (1977). Facial gestures which influence perception of status. Sociometry, 40, 374-378.

Keating, C., Mazur, A., \& Segall, M. (1981). A cross-cultural exploration of physiognomic traits of dominance and happiness. Ethology and Sociobiology, 2, 41-48.

Mazur, A., Halpern, C., \& Udry, J. R. (1994). Dominant looking male teenagers copulate earlier. Ethology and Sociobiology, $15,87-94$.

Milne, E., \& Grafman, J. (2001). Ventromedial prefrontal cortex lesions in humans eliminate implicit gender stereotyping.

Journal of Neuroscience, 21, 1-6.

Öngür, D., \& Price, J. L. (2000). The organization of networks within the orbital and medial prefrontal cortex of rats, monkeys, and humans. Cerebral Cortex, 10, 206-219.

Raleigh, M. J., \& Steklis, H. D. (1981). Effects of orbitofrontal and temporal neocortical lesions on the affiliative behavior of vervet monkeys. Experimental Neurology, 73, 378-389.
Reis, H. T., Wheeler, L., Spiegel, N., Kernis, M. H., Nezlek, J., Perri, M. (1982). Physical attractiveness in social interaction: Why does appearance affect social experience? Journal of Personality and Social Psychology, 43, 979-996.

Saver, J. L., \& Damasio, A. R. (1991). Preserved access and processing of social knowledge in a patient with acquired sociopathy due to ventromedial frontal damage.

Neuropsychologia, 29, 1241-1249.

Seltzer, B., \& Pandya, D. N. (1989). Frontal lobe connections of the superior temporal sulcus in the rhesus monkey. Journal of Comparative Neurology, 281, 97-113.

Senior, C., Barnes, J., Jenkins, R., Landau, S., Phillips, M. L., \& David, A. S. (1999). Attribution of social dominance and maleness to schematic faces. Social Behavior and Personality, 27, 331-337.

Shamay, S. G., Aharon-Peretz, J., Berger, B. D., \& Tomer, R. (2001). Impairment in cognitive and affective empathy in patients with prefrontal lesions: Correlations with cognitive performance. Brain and Cognition, 47, 353-356.

Stone, V. E., Baron-Cohen, S., \& Knight, R. T. (1998). Frontal lobe contributions to theory of mind. Journal of Cognitive Neuroscience, 10, 640-656.

Taylor, S. F., Liberzon, I., \& Koeppe, R. A. (2000). The effect of graded aversive stimuli on limbic and visual activation. Neuropsychologia, 38, 1415-1425. 